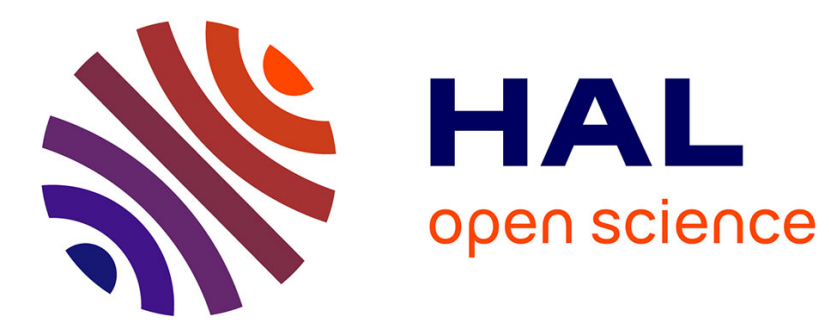

\title{
First vs second order magnetocaloric material for thermomagnetic energy conversion
}

Morgan Almanza, Alexander Pasko, Frédéric Mazaleyrat, Martino Lobue

\section{To cite this version:}

Morgan Almanza, Alexander Pasko, Frédéric Mazaleyrat, Martino Lobue. First vs second order magnetocaloric material for thermomagnetic energy conversion. IEEE Transactions on Magnetics, 2017, 10.1109/TMAG.2017.2697398 . hal-01514750

\section{HAL Id: hal-01514750 \\ https://hal.science/hal-01514750}

Submitted on 26 Apr 2017

HAL is a multi-disciplinary open access archive for the deposit and dissemination of scientific research documents, whether they are published or not. The documents may come from teaching and research institutions in France or abroad, or from public or private research centers.
L'archive ouverte pluridisciplinaire HAL, est destinée au dépôt et à la diffusion de documents scientifiques de niveau recherche, publiés ou non, émanant des établissements d'enseignement et de recherche français ou étrangers, des laboratoires publics ou privés. 


\title{
First vs second order magnetocaloric material for thermomagnetic energy conversion
}

\author{
Morgan Almanza ${ }^{1}$, Alexandre Pasko ${ }^{1}$, Frédéric Mazaleyrat ${ }^{1}$, Martino LoBue ${ }^{1}$ \\ ${ }^{1}$ SATIE, ENS Cachan, CNRS, Université Paris-Saclay, 94235 Cachan, France \\ morgan.almanza@ens-cachan.fr
}

\begin{abstract}
We estimate the power and efficiency of a thermal energy harvesting thermodynamic Brayton cycle using a first and second order magnetocaloric materials as active substance. The thermodynamic cycle was computed using a simple thermal exchange model and an equation of state deduced from a phenomenological Landau model. For the first and second order materials, narrow and high frequency cycles are optimum and give similar performances. Considering technological issues hindering the increase of frequency, we introduced a more detailed approach where we take into account the time needed to switch the material between two heat reservoirs. We show that the first order material equation of state leads thermodynamic cycle shape keeping it closer to the optimum cycle. Conditions to improve the performance of second order materials are discussed. In addition, we infer key remarks for prototype design regarding the power density and efficiency reachable in different configurations.
\end{abstract}

Index Terms-magnetocaloric materials, thermal energy harvesting, thermomagnetic cycle, simulation.

The supply of waste heat represents a huge and freely available amount of energy that makes it a key target for energy conversion technologies, notwithstanding the small thermodynamic efficiency to be expected because of the limited working temperature difference. Energy harvesting systems from waste heat based on thermomagnetic generation (TMG) have been studied since the 1948 paper by Brillouin and Iskenderian [1]. The new generation of magnetocaloric materials (MCM) raised a renewed interest towards this technology [2]. Recently we published numerical simulations of isofield-isotemperature and adiabatic-isotemperature cycles using a finite-time thermodynamics approach [3] (i.e. where thermal exchange is taken into account in a non-quasistatic regime). This allowed to estimate the efficiency at maximum power (EMP) using the first and second order phase transition magnetocaloric materials as active substance. Our preliminaries results made possible the comparison with thermoelectric generators [4]-[6], showing a similar power density for temperature span below $10^{\circ} \mathrm{C}$, but a much higher relative efficiency from 0.05 to 0.2 is attained in the case of thermomagnetic cycles. However, these cycles are still highly idealized as we assume a perfect control of their shapes based on field feedback. Indeed, the isotemperature transformations (i.e. a finite-time heat exchange where the temperature difference between the engine and the source is kept constant) used in [3] to work out best efficiency can be hardly achieved in an actual device that would more easily work on an isofieldadiabatic cycle (i.e. a Brayton cycle).

Here we will show how the constitutive relation (equation of state) of the material leads the actual shape of a finite-time thermodynamic cycle. Our main result is that in the first order MCM the isofield transformation stays closer to the isotemperature one allowing a significant efficiency improvement with respect to the second order MCM. Using the method presented in [3], we study the potential benefit of the first order MCM in terms of power density and efficiency as compared to the second order MCM for a cycle with adiabatic and isofield processes. In other words, we study how the shape of the cycle resulting from the state function of the MCM affects the efficiency at maximum power of the system.

\section{SYSTEM AND MATERIAL MODELING}

Two approaches are commonly envisaged to harvest the magnetic energy produced by cycling of the active material around a temperature induced ferromagnetic-paramagnetic transition. The first one uses the magnetization change in time to drive electric current [7], [8], whereas the second one uses the mechanical work associated with the difference of magnetic force due to magnetization change [9], [10]. Because of design constraints, a thermodynamic cycle composed by two isofield and two adiabatic processes (Brayton cycle) is a common choice for systems of the latter class.

Here we compute the thermodynamic cycle following [3], namely using an equation of state deduced from a phenomenological Landau model with magnetoelastic coupling [12] (for a review of similar approaches see also [11]). This model was developed to describe the first order phase transition in the $\mathrm{Mn}_{1.3} \mathrm{Fe}_{0.65} \mathrm{P}_{0.5} \mathrm{Si}_{0.5}$ magnetocaloric compound. The equation of state is presented in a dimensionless form, and the scale parameters connecting the internal variables with observed temperature, field and magnetization are roughly fitted to the real material. As for the second order transition, we use the fact that the magnetic behavior of this thermodynamic system is affected by the Landau coefficient related to strain. By modifying this coefficient, we can change the order of temperature-induced phase transition. Therefore, we slightly modify this parameters to be at the limit where we obtain a continuous transition i.e. a second order transition The obtained equation of state still may (or may not) correspond to a real material as, depending on the chemical composition, both first and second order transitions are observed in the Mn-Fe-P-Si system. In any case, this comparison is useful to emphasize the differences between sharp and smooth phase transitions. Because Landau model does not fit the total thermal capacity, a logarithmic term $C \ln (T)$ is added to the entropy to introduce a constant $C$ (lattice contribution) in the thermal capacity [3]. Here we use $C$ values similar to those encountered in most $\operatorname{MCM}\left(3.9 \mathrm{~J} \cdot \mathrm{K}^{-1} \cdot \mathrm{cm}^{-3}\right)$.

Figure 1 and Figure 2 show two examples of thermodynamic Brayton cycles, calculated using the equation of state of the first order MCM, represented in the $T(S)$ (temperature-entropy) and $M(H)$ (magnetization-field) planes, respectively. In Figure 2, 
during adiabatic magnetization (process 1), the cycle crosses the isotherms (thin lines) as the temperature of the MCM increases. Figure 3 shows an example of the second order MCM.

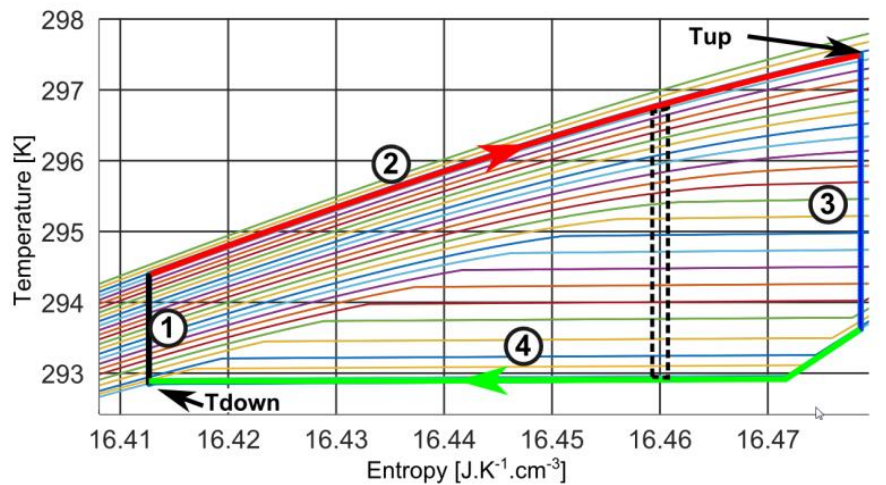

Figure 1. Two Brayton cycles on $T(S)$ diagram for the first order MCM with isofields in thin lines. Numbers from 1 to 4 indicate respectively the adiabatic magnetization, the isofield heat exchange with the hot reservoir, the adiabatic demagnetization, the isofield heat exchange with the cold reservoir.

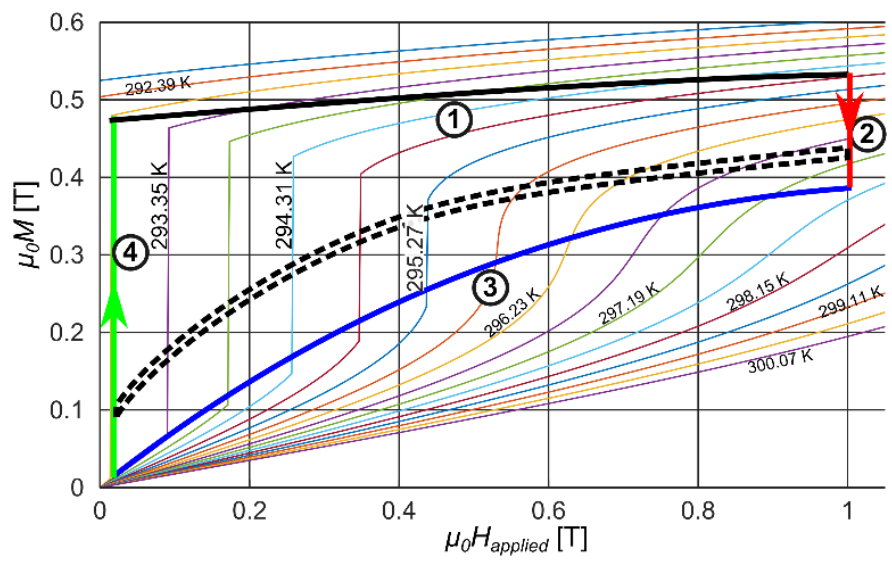

Figure 2. $M(H)$ cycle for a first order material. Thin lines represent isotherms from $291.91 K$ to $300.07 \mathrm{~K}$ with step of $0.48 \mathrm{~K}$. Numbers from 1 to 4 have the same meaning as in Fig. 1. The dashed thick lines represent a loaded cycle as described in section IV.

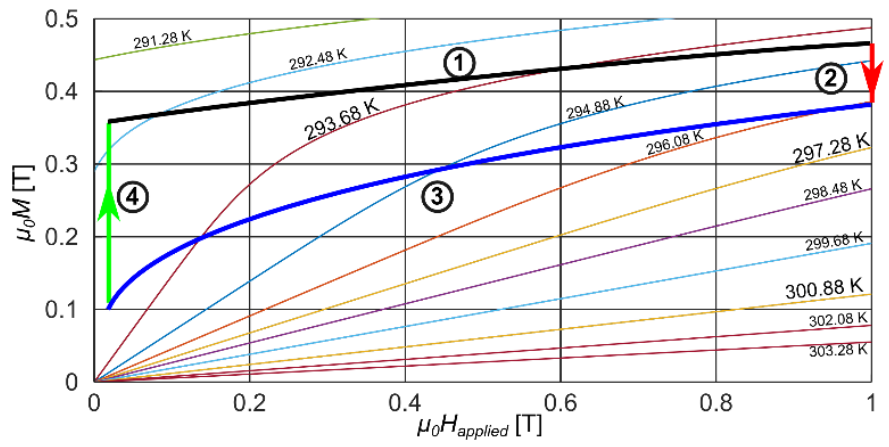

Figure 3. Cycle on $M(H)$ diagram for the second order MCM with isotherms from $291.28 \mathrm{~K}$ to $303.28 \mathrm{~K}$ with step of $1.2 \mathrm{~K}$, numbers 1 to 4 have the same meaning as in Fig. 1.

The MCM exchanges heat with reservoirs at the temperature $T_{\text {res }}$, where $T_{\text {res }}=T_{\text {hot }}$ or $T_{\text {res }}=T_{\text {cold }}$ when it is in contact with the heat source or with the heat sink, respectively. The heat exchange model, used to mimic the finite-time heat exchange, is given by

$$
\delta Q=\left[-k_{h i g h}\left(T-T_{\text {res }}\right)\right] d t
$$

where the heat exchange coefficient $k_{h i g h}$ is chosen as $1 \mathrm{~W}$. $\mathrm{cm}^{-3} \cdot \mathrm{K}^{-1}$. It is estimated by considering a $1 \mathrm{~mm}$ thickness sheet of MCM separated from the reservoir by an air layer of $25 \mu \mathrm{m}$ due to bad mechanical contact associated with surface roughness. The thermal conductance of a typical metallic MCM is much higher than the air gap conductance.

\section{THERMODYNAMIC SIMULATIONS}

Considering the MCM at thermodynamic equilibrium $\delta Q=$ $T d s$ and the equation of state $s(T, H)$, integration of (1) gives the time of exchange $t_{\text {exchange }}$ :

$$
t_{\text {exchange }}=\int_{\text {cycle }} d t=\int_{\text {cycle }} \frac{T d s}{\left[-k_{\text {high }}\left(T-T_{\text {res }}\right)\right]}
$$

In the first approximation we assume $t_{\text {period }}=t_{\text {exchange }}$, namely the time $t_{\text {switching }}$ spent along the adiabatic processes is assumed to be negligible. For example, if the temperature was fixed during the isofield process, then the time of exchange would be proportional to the entropy span. Therefore, the size of the cycle is correlated to the time of exchange, i.e. wider cycle has greater exchange time (dashed lines in Figure 2 show a tiny cycle associated with small $t_{\text {exchange }}$ ). Given the power density, the maximum relative efficiency $\eta_{\text {rel }}$, with respect to the Carnot efficient $\eta_{\text {Carnot }}$, is achieved by minimizing the entropy production $s_{i}$ as shown in the following equation:

$$
\eta_{\text {rel }}=\frac{\eta}{\eta_{\text {Carnot }}}=1-\frac{s_{i} T_{\text {cold }}}{\eta_{\text {Carnot }} Q_{\text {hot }}}
$$

where $Q_{\text {hot }}$ is the heat exchanged with the hot reservoir. Here we take into account only the entropy production associated with the finite-time heat exchange (i.e. the hysteresis and kinetics associated with the magnetic transition are neglected, a state of things often referred to as endoreversibility), which defines the degree of departures from thermal equilibrium. We have:

$$
\delta s_{i}(T, H)=\left(\frac{1}{T}-\frac{1}{T_{\text {res }}(H)}\right) \delta Q(T, H)
$$

Using the heat exchange model (1), the entropy produced is given by the following expression:

$$
s_{i}=k_{\text {high }} \int_{\text {tperiod }} \frac{\left(T_{\text {res }}(t)-T(t)\right)^{2}}{T_{\text {res }}(t) T(t)} d t
$$

therefore the efficiency and power are deduced from (3) and

$$
P=\eta_{\text {rel }} \eta_{\text {Carnot }} Q_{\text {hot }} / t_{\text {period }}
$$

It can be shown using (5) that $s_{i}$ is minimum when the heat exchange takes place at a constant temperature difference (HECTD). This means that efficiency is maximum when the difference between the temperature $T(t)$ of the MCM as a function of time $t$ and the temperature of the reservoirs, $T_{\text {hot }}$ or $T_{\text {cold }}$, respectively for the hot and cold reservoirs, are constant during the heat exchange process. That is why we use the term isotemperature rather than isotherm to name this process. 


\section{PARAMETERS AFFECTING THE POWER DENSITY AND THE EFFICIENCY}

A sound comparison between materials with the first and second order transitions needs a careful definition of the relevant parameters affecting the efficiency and the power density. We first define the maximum power conditions and eventually work out the efficiency at maximum power (EMP). In section IV we will argue that, when the period $t_{\text {exchange }}$ tends to zero, all the cycles approach the isotemperature and isofield ones, whatever the order of the transition. For this class of cycles we worked out an analytical expression in [3], allowing easy determination of the relevant parameters. The power will be considered negative using standard thermodynamic convention. Maximum power is achieved when thermodynamic cycles are centered between the temperatures of the reservoirs to ensure the same driving temperature difference $T-T_{\text {res }}$ during heat exchange processes. Using the results presented in [3], we write:

$$
\begin{gathered}
\eta_{\text {rel }}=\frac{\Delta T_{\text {adia }}}{\Delta T_{\text {res }}} \\
P\left(\eta_{\text {rel }}\right)=-\frac{\Delta T_{\text {res }}^{2} k_{\text {high }}}{4 T_{\text {hot }}} \eta_{\text {rel }}\left(1-\eta_{\text {rel }}\right)
\end{gathered}
$$

Here $\Delta T_{\text {adia }}\left(T, H_{f}, H_{i}\right)$, where $H_{f}$ and $H_{i}$ are the final and initial applied fields, depends on the equation of state [13] and $\Delta T_{\text {res }}=T_{\text {hot }}-T_{\text {cold }}$. Indeed, the maximum power is achieved when the temperature span of the reservoir is twice the adiabatic temperature change (i.e. $\Delta T_{\text {adia }}=\Delta T_{\text {res }} / 2$ ). As long as the field available is strong enough to reach this condition, the power increases proportionally to $\Delta T_{\text {res }}^{2}$ with

$$
P=-\frac{\Delta T_{\text {res }}^{2} k_{\text {high }}}{16 T_{\text {hot }}}
$$

keeping the relative efficiency around $50 \%$. This is a key technological issue inciting researches towards materials showing high $\Delta T_{\text {adia }}$ under low applied fields as in an actual device fields higher than $1 T$ can be hardly achieved. When the maximum field is reached, the system does not work anymore with the best cycle, i.e. with the maximum power cycle among all. The system works at its maximum power for its maximum $\Delta T_{\text {adia }}$ and the power increases proportionally to $\Delta T_{\text {res }}$ with

$$
P=-\frac{\left(\Delta T_{\text {res }}-\Delta T_{\text {adia }}\right) \Delta T_{\text {adia }} k_{\text {high }}}{4 T_{\text {hot }}}
$$

In addition, the EMP decreases as indicated in (7). These expressions are useful tools to estimate the harvested power. In the following, we numerically find the maximum power cycle keeping constant $\Delta T_{\text {adia }}$ and $\Delta T_{\text {res }}$. But we should be aware that for a given isotemperature, isofield cycle, an increase of $k_{\text {high }}$ will increase the performance (power density and/or efficiency) whereas an increase of temperature span $\Delta T_{\text {res }}$ will increase the power density but also decrease the efficiency unless accompanied by equal $\Delta T_{\text {adia }}$ increase. In our simulations, the applied field for the first and second order MCM is chosen in order to have the same maximum adiabatic temperature change $\Delta T_{\text {adia }}$
IV. CYCLE SHAPE FOR THE FIRST AND SECOND ORDER MATERIALS

In Figure 4 we show the calculated cycles with $T_{\text {hot }}=298 \mathrm{~K}$, $T_{\text {cold }}=291 \mathrm{~K}$. Full line cycles, corresponding to $t_{\text {exchange }}=$ $10 \mathrm{~s}$ (the wide cycles in the figure) give for first order $P_{m a g}=$ $-9.2 \mathrm{~mW} . \mathrm{cm}^{-3}$ and $\eta_{\text {rel }}=0.41$, and for second order $P_{\text {mag }}=-8.2 \mathrm{~mW} \cdot \mathrm{cm}^{-3}$ and $\eta_{\text {rel }}=0.41$. Tiny cycles, corresponding to $t_{\text {exchange }}=0.1 \mathrm{~s}$ (dashed line in the figure), give the same results for the first and second order MCM, namely $P_{\text {mag }}=-10 \mathrm{~mW} \cdot \mathrm{cm}^{-3}, \eta_{\text {rel }}=0.45$. Figure 4 shows that the first order MCM in isofield processes keeps closer to a HECTD (i.e. closer to the optimum cycle) than the second order MCM. The difference is apparent when comparing the low temperature isofield line in Figure 4, the first order material (red line, right) shows a horizontal line (a perfect isotemperature process) whereas the second order one (black line, left) shows a steeper slope. Results for the same $t_{\text {exchange }}$ show the same efficiency but not the same power density. Therefore, considering cycles with the same power density, the efficiency will be lower for the second order MCM as expected.

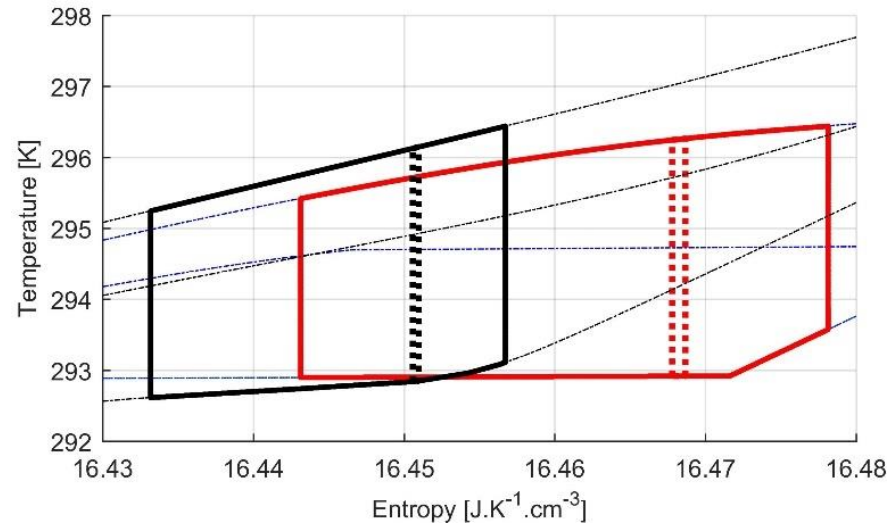

Figure 4. Brayton cycles on $T(S)$ diagram for the second order MCM (black) on the left and the first order MCM (red) on the right for large (solid line) and small cycles (dotted line). Thin lines represent three isofields in black for the second order MCM and in blue for the first order MCM.

Nevertheless, it is worth noting that both second and first order MCM can approach HECTD by drastically reducing the entropy span of the isofield process, as in the dashed line cycles in Figure 4. This means EMP for the first and second order materials will be very similar when working on tiny cycles at a rather high frequency. We shall use the term low loaded cycle to describe tiny cycles close to the HECTD. Indeed, only for a given heat exchange coefficient and temperatures of the reservoirs, low loaded cycles correspond to higher frequency than high loaded cycles.

\section{MAXIMUM POWER CYCLE FOR DIFFERENT EXCHANGE TIMES}

Low loaded cycles deal with small amount of energy including heat exchanged, that is why exchange time (2) is also small. It is not trivial to estimate the power density, especially as the cycles are not always close to isotemperature processes. An algorithm, based on simulations at constant reservoir temperatures, explores all possible cycles, i.e. all possible $T_{u p}$ and $T_{\text {down }}$, the temperatures of the ascending and descending adiabatics processes (as shown in Figure 1), to find the 
maximum power cycle for different $t_{\text {exchange. Results are }}$ shown in Figure 5 for the first and second order MCM. The choice of $t_{\text {exchange }}$ as a variable to study the power density will be discussed in the next section. In Figure 5 a slight increase of the power density for the first order MCM for longer periods is apparent. Cycles corresponding to exchange time of $0.1 \mathrm{~s}$ and $10 \mathrm{~s}$ are shown in Figure 4.

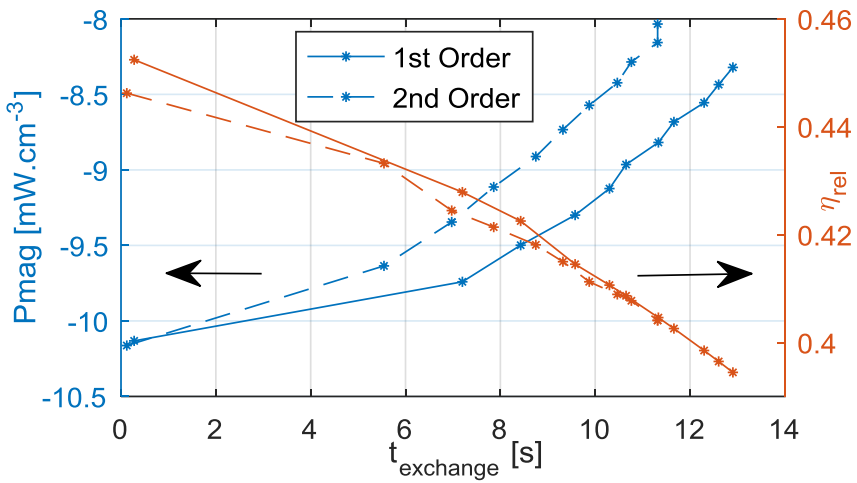

Figure 5. Maximum magnetic power and its relative efficiency for different exchange times for the first order in solid line $\left(H_{\text {applied }}=\right.$ $\left.0.67 T, \max \left(\Delta T_{\text {adia }}\right)=3.4 \mathrm{~K}\right)$ and for the second order in dashed line $\left(H_{\text {applied }}=1 \mathrm{~T}, \max \left(\Delta T_{\text {adia }}\right)=3.4 \mathrm{~K}\right)$ with $\Delta T_{\text {res }}=6.8 \mathrm{~K}, T_{\text {hot }}=298 \mathrm{~K}$ and $T_{\text {cold }}=291 \mathrm{~K}$.

As we argued in the previous section, both first and second order MCM show maximum power density and efficiency close to the optimum cycle for exchange time that tends to zero, i.e. for a low loaded cycles.

To emphasize the difference between the second and first order transitions, the adiabatic temperature change from now on is changed from 3 to $1.5 \mathrm{~K}$, accordingly $\Delta T_{\text {res }}$ is changed from 6.8 to $3.7 \mathrm{~K}$ in order to keep in same configuration. Power density and efficiency for this case are shown in Figure 6

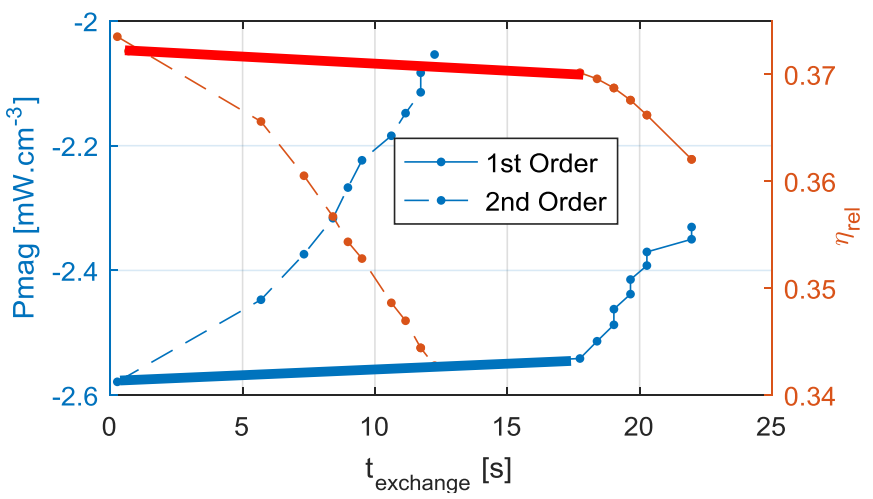

Figure 6. Maximum magnetic power and its relative efficiency for different time periods for the first order in solid line $\left(H_{\text {applied }}=0.28 T, \max \left(\Delta T_{\text {adia }}\right)=\right.$ $1.5 \mathrm{~K})$ and for the second order in dashed line $\left(H_{\text {applied }}=\right.$ $\left.0.33 \mathrm{~T}, \max \left(\Delta T_{\text {adia }}\right)=1.5 \mathrm{~K}\right)$ with $\Delta T_{\text {res }}=3.75 \mathrm{~K}, T_{\text {hot }}=295.53 \mathrm{~K}$ and $T_{\text {cold }}=291.8 \mathrm{~K}$.

A careful analysis of the results reveals some subtle details. The relative efficiency does not reach 0.5 because the system does not work exactly at $2 \Delta T_{\text {adia }}=\Delta T_{\text {res }}$, using (7) the relative efficiency is estimated around 0.4. For the first order MCM, where isofield lines are horizontal in the TS diagram, the power density and the efficiency are nearly constant whatever the exchange time (highlighted line in Figure 6). Because $T(H)$ isofield lines are not perfectly horizontal due to the lattice thermal capacity, the power and relative efficiency are slightly decreasing (highlighted line in Figure 6). Eventually, the relative efficiency does not exactly follow (7), because of not ideal isotemperature process.

\section{SWITCHING TIME}

In a real device the period $t_{\text {period }}$ of the cycle is the sum of the time spent to exchange heat $t_{\text {exchange }}$ (phases 2 and 4 in Figure 1) and the time spent along the two adiabatic branches (phases 1 and 3 in Figure 1), we refer to the latter as the switching time

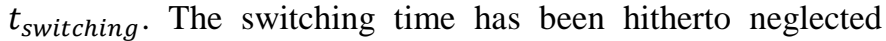
assuming $t_{\text {switching }} \ll t_{\text {exchange }}$ so that $t_{\text {exchange }} \sim t_{\text {period }}$. Now we would like to briefly discuss the case where the switching time cannot be neglected.

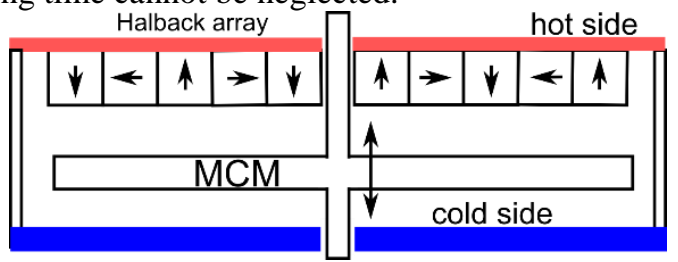

Figure 7. Example of thermomagnetic generator converting magnetization change to mechanical power (motion-force).

In Figure 7 we show a typical device to harvest the energy due to the difference of magnetic force during displacement between the hot and cold reservoirs [9]. If we assume $t_{\text {switching }} \sim 0$, the power transfer through the mechanical force and motion is infinite because the MCM passes between from one magnetic state to another in zero time, which is not physically possible. A finite switching time is needed to properly model the actual harvesting process. Therefore, we write $t_{\text {period }}=t_{\text {exchange }}+t_{\text {switching }}$. The power density being the area of the cycle divided by the period

$$
P=\frac{1}{t_{\text {period }}} \int_{\text {Cycle }} T d S
$$

for a fixed cycle, the power density depends only on $t_{\text {period }}$. Because in the previous part we considered $t_{\text {period }}=t_{\text {exchange }}$, we can deduce the power as a function of switching time by replacing the period $t_{\text {exchange }}$ with $t_{\text {exchange }}+t_{\text {switching }}$. Therefore, the previous power density has to be multiplied by the switching factor

$$
c_{\text {switching }}=\frac{t_{\text {exchange }}}{t_{\text {exchange }}+t_{\text {switching }}}
$$

Taking into account the switching time defines an upper bound to the maximum power that becomes relevant in the case of low loaded cycles where $t_{\text {exchange }}$ is vanishing and therefore $t_{\text {switching }}$ cannot be neglected.

Thin solid lines in Figure 8 show the power density as a function of $t_{\text {switching }}$, normalized to its low loaded cycle value, calculated for different fixed periods (i.e. fixed $t_{\text {exchange }}$ ). It is worth noting that, in this case, normalizing to the low loaded cycle power makes the first and second order lines collapse on the same curve. In the same figure we show the maximum normalized power density as a function of the switching time (dashed lines). In this case, our algorithm selects the maximum power cycle given $t_{\text {switching }}$ (dashed line in Figure 8) by 
changing the exchange time; namely, each point on the dashed lines corresponds to a cycle with different period using power maximization as a constraint. The set of cycles found for different switching times considered, limits the decrease of the power density as shown by dashed line in Figure 8 .

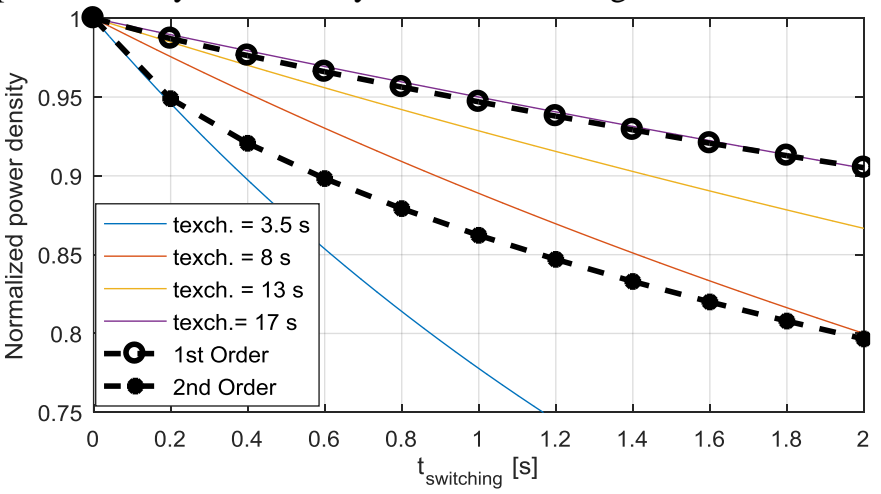

Figure 8. Effect of the switching time on the power density normalized with respect to the low loaded cycle, with $\Delta T_{\text {adia }}=1.5 \mathrm{~K}$. Dashed lines are the

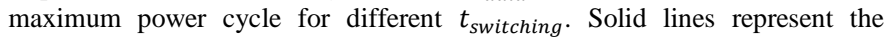
normalized power density for a fixed $t_{\text {exchange }}$.

The power maximization algorithm tends to compensate the switching time decrease by selecting cycles with larger $t_{\text {exchange }}$ (i.e. larger periods, as shown in Figure 9). However, as discussed in the previous sections, larger $t_{\text {exchange }}$ is getting the isofield transformations away from the isotemperature curves drastically reducing the cycle relative efficiency for the second order MCM.

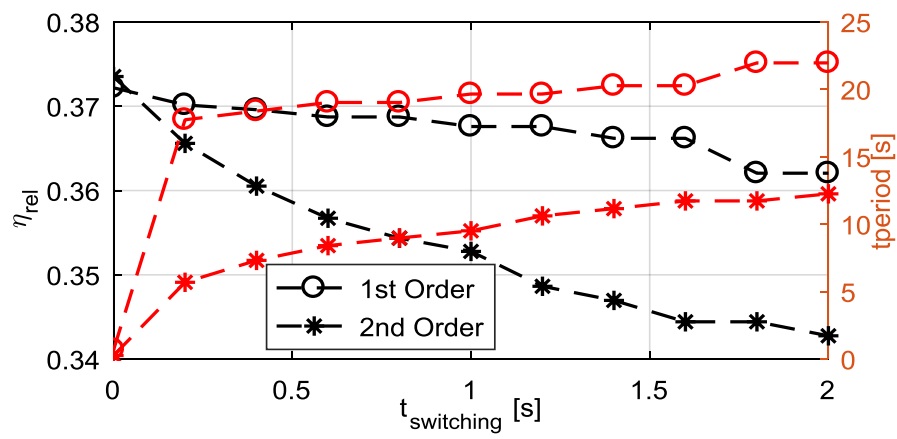

Figure 9 Relative efficiency (black) and period (red) of the maximum power cycle for different $t_{\text {switching }}$ with $\Delta T_{\text {adia }}=1.5 \mathrm{~K}$ for the first order (circles) and for the second order (asterisks).

In Figure 10, maximum power cycles for $t_{\text {switch }} \sim 0$ and $0.8 \mathrm{~s}$ show that the first order cycles are closer to HECTD compared to the second order ones when $t_{\text {switch }}$ equals $0.8 \mathrm{~s}$.

This is a key point to compare the first and second order MCM performances. First order materials can increase the $t_{\text {exchange }}$ without reducing significantly the efficiency of the cycle, because they keep closer to HECTD (Figure 10). As shown in Figure 8 and Figure 9, EMP and power density of the first order MCM are less sensitive to the effect of increasing $t_{\text {switching }}$.

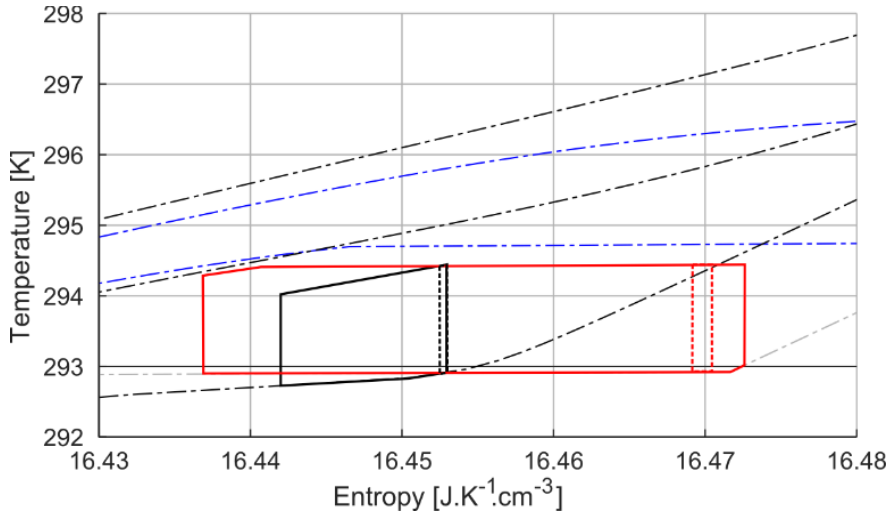

Figure 10. Maximum power Brayton cycles for $t_{\text {switch }} \sim 0$ i.e. small cycles (dotted line) and $t_{\text {switch }}=0.8 \mathrm{~s}$ i.e. large cycles (solid line) on $T(S)$ diagram

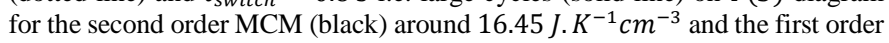
MCM (red) around $16.47 \mathrm{~J} . \mathrm{K}^{-1} \mathrm{~cm}^{-3}$. Thin lines represent three isofields in black for second order and in blue for first order.

\section{CONCLUSION}

This study evaluates the benefit of using MCM with high $\Delta T_{a d i a}$ and systems with high heat exchange coefficient $k_{\text {high }}$ for thermomagnetic energy conversion. Our simulations reveal that low loaded cycles are beneficial in terms of efficiency and power density but involve an increase of the operating frequency. At low frequency, the time taken along the adiabatic processes can be neglected. Because of the frequency increase and considering technical constraints, the model needs to take into account the time to switch between the reservoirs, called the switching time.

The first order MCM shows its robustness to the introduction of the switching time as compared to the second order MCM in terms of power and relative efficiency. An increase of the heat exchange coefficient $k_{\text {high }}$ paves the way to potentially much higher power densities, due to a decrease of $t_{\text {exchange }}$. However, in this case, the reduction of the switching time becomes a key technological issue.

In section $\mathrm{V}$ we did show that, when the switching time can be neglected, the first and second order MCM may show similar performances only in the case of low loaded cycles when $t_{\text {exchange }}$ becomes small. However, in section VI we argued that in low loaded cycle case $t_{\text {switching }}$ can hardly be neglected and, also in this case, the power density at maximum efficiency of the second order materials is drastically reduced, with respect to the first order ones, when switching time is relevant.

However, high frequency excitations will very possibly increase the role of transition kinetics (neglected in our approach) and tiny thermodynamic cycles will be more affected by hysteresis. Both effects have been neglected in our discussion and can be relevant in the case of the first order MCM.

Small period cycles are expected to be achievable mostly in micro-systems where a fast heat exchange and a fast switching are expected. We can conclude that, while the first order materials are definitely better suited for bulk applications, further investigations must be devoted to the case of highfrequency micro-systems, taking into account the possible shortcomings of the first order materials associated with the role of non-equilibrium phenomena in the phase transitions. 


\section{ACKNOWLEDGMENT}

This work has benefited from the financial support of the LabeX LaSIPS (ANR-10-LABX-0040-LaSIPS) managed by the French National Research Agency under the "Investissements d'avenir" program ( ${ }^{\circ}$ ANR-11-IDEX-000302), project MaETEC.

\section{REFERENCES}

[1] L. Brillouin and H. P. Iskenderian, "Thermomagnetic generator," Fed. Telecommun. Lab., 1948.

[2] D. Vuarnoz et al., "Quantitative feasibility study of magnetocaloric energy conversion utilizing industrial waste heat," Appl. Energy, vol. 100, pp. 229-237, Dec. 2012.

[3] M. Almanza, A. Pasko, F. Mazaleyrat, and M. LoBue, "Numerical study of thermomagnetic cycle," J. Magn. Magn. Mater., vol. 426, pp. 64-69, Mar. 2017.

[4] M. Almanza, A. Pasko, A. Bartok, F. Mazaleyrat, and M. Lobue, "Thermal energy harvesting: thermomagnetic versus thermoelectric generator," in 7th International Conference on Magnetic Refrigeration at Room Temperature (Thermag VII), Turin, Italy, 2016.

[5] A. Petucco, S. Saggini, L. Corradini, and P. Mattavelli, "Analysis of Power Processing Architectures for Thermoelectric Energy Harvesting," IEEE J. Emerg. Sel. Top. Power Electron., vol. 4, no. 3, pp. 1036-1049, Sep. 2016.

[6] J. P. Carmo, L. M. Goncalves, and J. H. Correia, "Thermoelectric Microconverter for Energy Harvesting Systems," IEEE Trans. Ind. Electron., vol. 57, no. 3, pp. 861-867, Mar. 2010.

[7] T. Christiaanse and E. Brück, "Proof-of-Concept Static Thermomagnetic Generator Experimental Device," Metall. Mater. Trans. E, vol. 1, no. 1, pp. 36-40, Mar. 2014.

[8] M. Ohkoshi, H. Kobayashi, T. Katayama, M. Hirano, and T. Tsushima, "Rotational-type spin reorientation in Nd1-xDyxCo5 and its application to thermomagnetic generator," IEEE Trans. Magn., vol. 13, no. 5, pp. 1158-1160, Sep. 1977.

[9] C.-J. Hsu, S. M. Sandoval, K. P. Wetzlar, and G. P. Carman, "Thermomagnetic conversion efficiencies for ferromagnetic materials," J. Appl. Phys., vol. 110, no. 12, p. 123923, Dec. 2011.

[10] Chin-Chung Chen, Tien-Kan Chung, Chia-Yuan Tseng, Chiao-Fang Hung, Po-Chen Yeh, and Chih-Cheng Cheng, "A Miniature MagneticPiezoelectric Thermal Energy Harvester," IEEE Trans. Magn., vol. 51, no. 7, pp. 1-9, Jul. 2015.

[11] J.-C. Tolédano and P. Tolédano, The Landau theory of phase transitions: application to structural, incommensurate, magnetic and liquid crystal systems. Singapore: World Scientific, 1987.

[12] A. Pasko, A. Bartok, K. Zehani, L. Bessais, F. Mazaleyrat, and M. LoBue, "X-ray diffraction analysis of the magnetoelastic phase transition in the Mn-Fe-P-Si magnetocaloric alloy," AIP Adv., vol. 6, no. 5, p. 056204, May 2016.

[13] A. Smith, C. R. H. Bahl, R. Bjørk, K. Engelbrecht, K. K. Nielsen, and N. Pryds, "Materials Challenges for High Performance Magnetocaloric Refrigeration Devices," Adv. Energy Mater., vol. 2, no. 11, pp. 12881318, Nov. 2012. 